\title{
Suppression of Inflammation, Osteoclastogenesis and Bone Loss by PZRAS Extract
}

\author{
${\text { Liang } \mathrm{Li}^{1 \dagger} \text {, Young-Ran Park }}^{1 \dagger}$, Saroj Kumar Shrestha ${ }^{1}$, Hyoung-Kwon Cho ${ }^{2}$, and Yunjo Soh ${ }^{1,3 *}$ \\ 'Department of Dental Pharmacology, School of Dentistry, Jeonbuk National University, Jeonju 54896, Republic of Korea \\ ${ }^{2}$ Hanpoong Pharm and Foods Co., Ltd., Jeonju 561-841, Republic of Korea \\ ${ }^{3}$ Department of Pharmacology, School of Pharmacy and Institute of New Drug Development, Jeonbuk National \\ University, Jeonju 54896, Republic of Korea
}

Panax ginseng has a wide range of activities including a neuroprotective effect, skin protective effects, enhanced DNA repairing, anti-diabetic activity, and protective effects against vascular inflammation. In the present study, we sought to discover the inhibitory effects of a mixture of natural products containing Panax ginseng, Ziziphus jujube, Rubi fructus, Artemisiae asiaticae and Scutellaria baicalensis (PZRAS) on osteoclastogenesis and bone remodeling, as neither the effects of a mixture containing Panax ginseng extract, nor its molecular mechanism on bone inflammation, have been clarified yet. PZRAS upregulated the levels of catalase (CAT), superoxide dismutase (SOD), glutathione reductase (GSH-R) and glutathione peroxidase (GSH-Px) and reduced malondialdehyde (MDA) in LPS-treated RAW264.7 cells. Moreover, treatment with PZRAS decreased the production of IL-1 $\beta$ and TNF- $\alpha$. PZRAS also inhibited osteoclast differentiation through inhibiting osteoclastspecific genes like MMP-2, 9, cathepsin K, and TRAP in RANKL-treated RAW264.7 cells. Additionally, PZRAS has inhibitory functions on the RANKL-stimulated activation of ERK and JNK, which lead to a decrease in the expression of NFATc1 and c-Fos. In an in vivo study, bone resorption induced by LPS was recovered by treatment with PZRAS in bone volume per tissue volume (BV/TV) compared to control. Furthermore, the ratio of eroded bone surface of femurs was significantly increased in LPStreated mice compared to vehicle group, but this ratio was significantly reversed in PZRAS-treated mice. These results suggest that PZRAS could prevent or treat disorders with abnormal bone loss.

Keywords: PZRAS, antioxidant, anti-inflammatory, osteoclastogenesis, bone loss

Received: April 7, 2020 Accepted: August 12, 2020

First published online: August 15, 2020

*Corresponding author Phone: +82-63-270-4038 Fax: +82-63-270-4037 E-mail:ysoh@jbnu.ac.kr

${ }^{\dagger}$ These authors contributed equally to this work.

pISSN 1017-7825 elSSN 1738-8872

Copyright(C) 2020 by The Korean Society for Microbiology and Biotechnology

\section{Introduction}

Osteoporosis, characterized by low bone mass and destruction of bone microarchitecture, is a complicated disorder with multiple factors including estrogen deficiency, the aging process, and genetics, and leads to fracture risk predominantly in the older population [1]. Anti-bone resorptive drugs such as bisphosphonates, with their excellent bone-improving effects, have been extensively used [2]. However, many previous studies have reported that some of these drugs are also associated with various diseases including endo-metritis, thromboembolism, breast cancer, hypercalcemia, atrial fibrillation, and osteonecrosis of the jaw [3-5].

Natural flavonoid compounds are good resources for therapeutic agents because they do not carry severe risk along with the possibility of long-term treatment. Therefore, the selection of natural compounds is important in developing drugs for osteoporosis [7]. Many plants have been incorporated into domestic medicines and have anti-inflammatory and pharmacological properties ascribed to antioxidant compounds $[6,8]$.

During inflammation, reactive oxygen species (ROS) are produced to defend against infection $[9,10]$. Especially, excessive nitric oxide (NO) abnormally released by inducible nitric oxide synthase (iNOS) can damage macromolecules such as proteins, DNA, and lipids [11]. In recent reports, free radicals were found to be elevated in response to osteoclastic activity and lipid peroxidation [12]. In addition, the reduction of the antioxidant condition was manifested by low levels of glutathione peroxidase (GSH-Px), glutathione reductase (GSH-R) and superoxide dismutase (SOD) [13]. Matrix metalloproteinases (MMPs) are a group of proteinase-related enzymes that degrade the extracellular matrix. Increased production of MMPs is associated with chronic inflammatory diseases such as rheumatoid arthritis and periodontitis [14-18].

Some recent studies have reported that lipopolysaccharide (LPS)-stimulated osteoclastogenesis exacerbates bone destruction $[19,20]$. LPS plays an essential role in bone resorption, which includes assembling inflammatory cells, production of cytokines such as interleukin- $1 \beta$ (IL-1 $\beta$ ) and tumor necrosis factor- $\alpha$ (TNF- $\alpha$ ), and induction of osteoclastic differentiation [21]. Therefore, it is a beneficial approach to treat various disorders by reducing 
oxidative stress and inflammation processes.

Panax ginseng is a traditional herbal medicine employed as a general health-promoting tonic. Ginsenoside-Rg2 is the main active component that can be extracted from the root and stem leaves of $P$. ginseng. Additionally, ginsenoside-Rg2 has been known to act through a variety of mechanisms to mediate a neuroprotective effect, a skin protective effect, enhanced DNA repair, anti-diabetic activity and a protective effect against vascular inflammation [22, 23]. Ziziphus jujube has been used as herbal medicine and to cure oxidative stress-related diseases such as tumors and cardiovascular diseases [24]. Recently, goshonoside-F5 from Rubi fructus was reported to inhibit the Nuclear factor-кappa $B(N F-\kappa B)$ and MAPK signaling pathways [25]. The extract of Astragalus membranaceus has displayed anti-inflammatory and antioxidant effects in vitro [26]. Some compounds from Artemisia argyi supply a new lead compound for inflammatory disorders [27]. Z. jujube and Scutellaria baicalensis were known to have potential bone-forming effects [28]. However, there has been little investigation into the effect of mixed medicinal plant extracts containing $P$. ginseng on bone lytic disorders.

Here, we explored the antioxidant, anti-inflammatory, and bone protective effects of mixed extracts of P. ginseng, Z. jujube, R. fructus, A. asiaticae and S. baicalensis (PZRAS) in vitro and in mice.

\section{Materials and Methods \\ PZRAS Preparation Process}

The plant extracts were prepared, organized and purified by Hanpoong Pham \& Foods Co., Ltd. ( Korea). Briefly, $P$. ginseng was extracted with $60 \%$ ethanol while Z. jujube, R. fructus, A. asiaticae, and S. baicalensis were extracted with $30 \%$ ethanol, respectively. P. ginseng, Z. jujube, A. asiaticae, and S. baicalensis were obtained from Dong Kyung Pharm Co., Ltd. (Korea) and R. fructus was purchased from Omniherb Co., Ltd. (Korea). Each sample solution was separated through a $5 \mu \mathrm{m}$ membrane filter, evaporated under reduced pressure and dried at $70-80^{\circ} \mathrm{C}$. For experiment, powdered samples were weighed and mixed at a mass ratio of 4:1:1:1:1 (w/w). DMSO was used to dissolve PZRAS, which was then diluted in medium for cell culture as needed.

\section{Cell Culture and Osteoclast Differentiation}

RAW264.7 cells from American Type Culture Collection (ATCC, USA) were cultured with Dulbeccos Modified Eagles Media (DMEM) supplemented with 10\% Fetal Bovine Serum (FBS) and 100 units/ml penicillin and $100 \mu \mathrm{g} / \mathrm{ml}$ streptomycin at $37^{\circ} \mathrm{C}$ in $95 \%$ humidity and $5 \% \mathrm{CO}_{2}$. For osteoclast differentiation, RAW264.7 cells $\left(3 \times 10^{3}\right.$ cells/96 wells) were incubated with $50 \mathrm{ng} / \mathrm{ml}$ Receptor activator of nuclear factor kappa-B ligand (RANKL) with or without PZRAS for 6 days and stained using the Tartrate-resistant acid phosphatase (TRAP) staining kit (Sigma-Aldrich, USA).

\section{2,2-Diphenyl-1-Picrylhydrazyl (DPPH) Antioxidant Assay}

The DPPH assay method was performed to measure antioxidant potential of PZRAS according to the manufacturer's protocol. PZRAS was mixed with $0.1 \mathrm{M}$ of DPPH in culture plates and maintained at $37^{\circ} \mathrm{C}$ during $30 \mathrm{~min}$. DPPH radical scavenging activity of PZRAS was calculated by the following formula:

DPPH radical scavenging activity $(\%)=\left[1-A_{\text {sample }(540 \mathrm{~nm})} / A_{\text {control }(540 \mathrm{~nm})}\right] \times 100$

\section{MTT Assay}

MTT (3-(4, 5-dimethylthiazolyl-2)-2, 5-diphenylthtrazolium bromide) was used to evaluate cell viability [29]. Cells $\left(2 \times 10^{4}\right.$ cells/well) were stimulated with or without PZRAS for $24 \mathrm{~h}$, and incubated with $100 \mu \mathrm{g} / \mathrm{ml}$ of MTT reagent in each well for $2 \mathrm{~h}$ further. For cell viability, the optical density (OD) of the solution was recorded at $540 \mathrm{~nm}$ using a microplate reader (Molecular Devices, USA).

\section{Lipid Peroxidation (MDA) and Enzyme Activity Assays}

Thiobarbituric acid (TBA) was used to determine the amount of lipid peroxidation as previously identified [30]. The absorbance of solution was read at $532 \mathrm{~nm}$ and calculated based on the $\varepsilon$ value of 153,000.

Catalase (CAT) activity was measured by Aeby's method [31]. Superoxide dismutase (SOD) and GSH-Px activity were determined using an SOD determination kit (Oxis Research, USA) and a commercially available kit (Sigma-Aldrich) according to the manufacturers' guidance, respectively.

\section{Western Blot Analysis}

Western blotting was carried out by previously reported method [32]. In brief, whole cell lysates were obtained using radioimmuno-precipitatiotion assay (RIPA) buffer (iNtRON Biotechnology, Korea) containing $1 \mathrm{mM}$ phenylmethylsulfonylfluoride (PMSF), and $1 \times$ protease inhibitor cocktail. Proteins were separated using SDSPAGE and transferred subsequently onto PVDF membrane (Bio-Rad, USA) through a wet transfer system at 100 $\mathrm{V}$ and $350 \mathrm{~mA}$. The protein bands were detected using chemiluminescence (ECL Plus Kit, Amersham Biosciences, USA) and $\beta$-actin typically was utilized as a loading control.

\section{Enzyme-Linked Immunosorbent Assay (ELISA)}

To assess the secreted levels of pro-inflammatory cytokine (TNF- $\alpha$ and IL-1 $\beta$ ), cells were pre-treated with PZRAS for $30 \mathrm{~min}$ and stimulated by LPS $(2 \mu \mathrm{g} / \mathrm{ml})$ for $8 \mathrm{~h}$ and $24 \mathrm{~h}$, respectively. TNF- $\alpha$ and IL-1 $\beta$ ELISA kits (R\&D Systems, USA) were used to measure concentrations of cytokines in culture supernatants. 


\section{Reverse Transcription-Polymerase Chain Reaction (RT-PCR)}

According to the manufacturer's instructions, total RNA was extracted using TRI ${ }^{\text {zol }}$ reagent (Invitrogen, USA). SuperScript First-Strand Synthesis System (Invitrogen) was used to synthesize cDNA and the following primers were designed by Bioneer (Korea) for amplification: MMP-2, sense strand $5^{\prime}$-ggctggaacactaggac- $3^{\prime}$, antisense strand 5' -cgatgccatcaaagacaatg-3' (product size 289 bp); MMP-9, sense strand 5' -cgtcgtgatccccacttact- $3^{\prime}$, antisense strand $5^{\prime}$-tcctgggcaagcagtactct- $3^{\prime}$ (product size $433 \mathrm{bp}$ ); TRAP, sense strand $5^{\prime}$-ctgctgggcctacaaatcat- $3^{\prime}$, antisense strand 5' -ggtagtaagggctggggaag- $3^{\prime}$ (product size $400 \mathrm{bp}$ ); Cathepsin K, sense strand $5^{\prime}$-aggcggctatatgaccactg- $3^{\prime}$, antisense strand $5^{\prime}$-ccgagccaagagagcatatc- $3^{\prime}$ (product size $403 \mathrm{bp}$ ); c-Fos, sense strand $5^{\prime}$-atgggctctcctgtcaacac- $3^{\prime}$, antisense strand $5^{\prime}$-ggctgccaaaataaactcca- $3^{\prime}$ (product size $480 \mathrm{bp}$ ); NFATc1, sense strand $5^{\prime}$-gggtcagtgtgaccgaagat- $3^{\prime}$, antisense strand $5^{\prime}$-aggtgggtgaagactgaagg- $3^{\prime}$ (product size $280 \mathrm{bp}$ ); and $\beta$-actin, sense strand $5^{\prime}$-agaaaatctggcaccacacc$3^{\prime}$, antisense strand $5^{\prime}$-ccatctcttgctcgaagtcc- $3^{\prime}$ (product size $435 \mathrm{bp}$ ). After PCR reaction, PCR products were separated using 1.5-2.0 \% agarose gel electrophoresis with $0.5 \times$ TAE buffer and confirmed by comparison to a GeneRuler 100 bp DNA Ladder Plus (Fermentas, Canada).

\section{Lipopolysaccharides (LPS)-Induced Bone Resorption}

Animal experiments were performed under the guidance of the Jeonbuk National University Laboratory Animal Center (Approval no. CBNU 2018-094, Korea). Previous studies revealed that LPS leads to inflammatory bone resorption by direct intraperitoneal injection in mice as a bone erosion animal model [33]. Briefly, six-weekold ICR mice were injected intraperitoneally on days $1,4,7,11$ with or without LPS ( $5 \mathrm{mg} / \mathrm{kg}$ body weight). PZRAS $(100,200 \mu \mathrm{g} / \mathrm{kg}$ and $400 \mu \mathrm{g} / \mathrm{kg}$ of body weight) was administered orally 1 day prior to LPS injection and every day thereafter for 14 days. Six week-old male ICR mice used during the experiments were obtained from Damool Science (Korea).

\section{Microcomputed Tomographic Bone Analysis}

The femurs on the left sides of the mice were scanned with a 1076 Micro CT System (Skyscan, Belgium), as previously described [34]. A three-dimensional image reconstruction analysis system was used to assess bone volume / tissue volume fraction (BV/TV, \%).

\section{Histological Bone Analysis}

Paraffin section and staining preparation were determined as described previously [32]. The femurs of the other side were processed through fixation, decalcification, embedding, sectioning, and H\&E staining. Images were then obtained by a color digital video camera (ZEISS, West Germany) at $25 \times$ and $100 \times$ magnifications.

\section{Statistical Analysis}

All the experiments were repeated at least three times and expressed as means \pm S.D., unless otherwise indicated. Statistical analyses were performed using SPSS ver. 12.0 software, and $P$-values less than 0.05 were considered statistically significant.

\section{Results}

\section{Effect of PZRAS on Cell Viability of Mouse Macrophage RAW264.7 cells}

The cytotoxicity of PZRAS was evaluated by MTT assay. RAW264.7 cells were seeded and incubated with or without PZRAS $(0,0.1,1,10$, and $50 \mu \mathrm{g} / \mathrm{ml})$ for $24 \mathrm{~h}$. PZRAS showed no significant cytotoxicity compared with control group (Fig. 1).

\section{Effect of PZRAS on DPPH Radical Scavenging Activity}

DPPH radical scavenging analysis was extensively used to measure the antioxidant effect of biological samples [35]. The radical scavenging activity of PZRAS was compared with vitamin C as the standard antioxidant. As

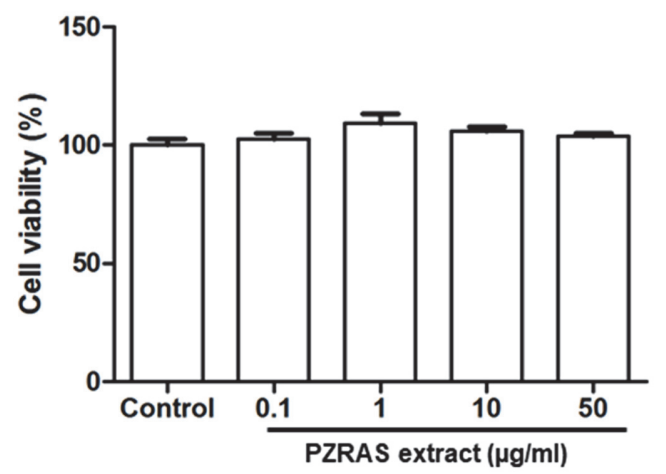

Fig. 1. Effect of PZRAS on cell viability. RAW264.7 cells were seeded for $16 \mathrm{~h}$, and then treated with various concentrations $(0.1,1,10$, and $50 \mu \mathrm{g} / \mathrm{ml})$ of PZRAS for $24 \mathrm{~h}$. Cell viability was determined as described in Materials and Methods. The values determined the means \pm SD of at least three independent experiments. 
Table 1. Radical scavenging activities of PZRAS and vitamin C.

\begin{tabular}{lc}
\hline & $\mathrm{IC}_{50}(\mu \mathrm{g} / \mathrm{ml})$ \\
\hline PZRAS & $16.0 \pm 1.4$ \\
Vitamin C & $10.4 \pm 2.1$ \\
\hline
\end{tabular}

Table 2. Effects of PZRAS on antioxidant enzyme activities in LPS-induced RAW264.7 cells.

\begin{tabular}{lcccccc}
\hline & Untreated & \multicolumn{5}{c}{ PZRAS $(\mu \mathrm{g} / \mathrm{ml})+\mathrm{LPS}(2 \mu \mathrm{g} / \mathrm{ml})$} \\
\hline & & 0 & 0.1 & 1 & 10 & 50 \\
\hline${ }^{\mathrm{a}}$ CAT (Units/mg Protein) & $3.78 \pm 0.80$ & $3.90 \pm 0.03^{*}$ & $3.88 \pm 0.02$ & $4.01 \pm 0.04$ & $4.43 \pm 0.05^{*}$ & $4.46 \pm 0.04^{\star}$ \\
${ }^{\mathrm{b}} \mathrm{SOD}$ (Units/mg Protein) & $46.9 \pm 1.90$ & $61.6 \pm 4.80^{*}$ & $65.7 \pm 7.40$ & $77.9 \pm 3.60$ & $98.0 \pm 3.70^{*}$ & $108.1 \pm 2.80^{*}$ \\
${ }^{\mathrm{c}} \mathrm{GSH}-\mathrm{Px}(\mathrm{mU} / \mathrm{ml}$ Protein) & $36.14 \pm 0.49$ & $25.3 \pm 0.44$ & $28.84 \pm 1.00$ & $33.9 \pm 0.92^{*}$ & $26.72 \pm 0.13$ & $19.36 \pm 0.28$ \\
${ }^{\mathrm{d}}$ GSH-R $(\mu \mathrm{mol} /$ L Protein) & $622.9 \pm 24.80$ & $448.5 \pm 6.60^{\#}$ & $520.2 \pm 28.50$ & $566.1 \pm 13.90^{*}$ & $420.3 \pm 20.10$ & $390.1 \pm 25.20$
\end{tabular}

${ }^{\#} p<0.05$ as compared with the untreated group. ${ }^{*} p<0.05$ as compared with the LPS-treated group only.

a. catalase; $b$. superoxide dismutase; $c$. glutathione peroxidase; d. glutathione reductase

shown in Table 1, PZRAS exhibited radical scavenging activity with an $\mathrm{IC}_{50}$ of $16.0 \pm 1.4 \mu \mathrm{g} / \mathrm{ml}$, whereas vitamin C showed $10.4 \pm 2.1 \mu \mathrm{g} / \mathrm{ml}$. Therefore, PZRAS has a radical scavenging activity comparable to vitamin C.

\section{Effects of PZRAS on Antioxidant Enzymes}

To confirm whether PZRAS exhibited an antioxidant activity, activities of CAT, SOD, GSH-R, and GSH-Px and MDA level were measured. As shown in Table 2, CAT activity was significantly enhanced by a $10 \mu \mathrm{g} / \mathrm{ml}$ concentration of PZRAS $(4.43 \pm 0.05 \mathrm{units} / \mathrm{mg})$ as well as in a $50 \mu \mathrm{g} / \mathrm{ml}(4.46 \pm 0.04 \mathrm{units} / \mathrm{mg})$ concentration compared with LPS-treated group (3.90 $\pm 0.03 \mathrm{units} / \mathrm{mg}$ ). Moreover, the activity of SOD significantly increased in 10 and $50 \mu \mathrm{g} / \mathrm{ml}$ PZRAS $(98.0 \pm 3.7$ and $108.1 \pm 2.8$ units $/ \mathrm{mg})$ concentrations compared with the LPS-alone group (61.6 \pm 4.8 units/mg). PZRAS treatment markedly suppressed GSH-Px and GSH-R in LPS-induced RAW264.7 cells. However, GSH-Px and GHS-R activity significantly increased in concentration of $1 \mu \mathrm{g} / \mathrm{ml}$ PZRAS $(33.9 \pm$ 0.92 and $566.1 \pm 13.90$ units/mg, respectively). The MDA level was higher than control in LPS-stimulated RAW264.7 cells, whereas PZRAS markedly reduced MDA level in 10 and $50 \mu \mathrm{g} / \mathrm{ml}$ concentrations compared with LPS-only group (Fig. 2). The results suggest that high doses $(10$ and $50 \mu \mathrm{g} / \mathrm{ml}$ ) of PZRAS are more potent than low doses and PZRAS exhibits effective antioxidant capacity in LPS-treated RAW 264.7 cells.

\section{Effect of PZRAS on LPS-Induced Pro-Inflammatory Cytokines}

In order to explore the anti-inflammatory effect of PZRAS, RAW264.7 cells were treated with or without PZRAS. As shown in Fig. 3A, the stimulation with LPS increased the iNOS protein expression in RAW264.7 cells. However, $50 \mu \mathrm{g} / \mathrm{ml}$ of PZRAS downregulated LPS-induced iNOS protein level compared with LPS-only group $(p<0.05)$.

Generally, TNF- $\alpha$ and IL- $\beta$ are released by LPS-stimulated macrophage cells and are associated with increased inflammatory responses [36]. To better perceive the effect of PZRAS on inflammation, secreted levels of TNF- $\alpha$ and IL- $\beta$ in the cultured medium of RAW 264.7 cells were measured by ELISA. TNF- $\alpha$ level was markedly upregulated by LPS treatment, but PZRAS at $50 \mu \mathrm{g} / \mathrm{ml}$ concentration significantly decreased this induction $(p<$ $0.05)$ (Fig. 3B). The level of IL- $1 \beta$ was increased by LPS treatments, and this increased level of IL- $1 \beta$ was strongly suppressed by PZRAS (Fig. 3C). These results indicated that PZRAS can manipulate the LPS-induced proinflammatory activity of cytokine, TNF- $\alpha$ and IL- $\beta$.

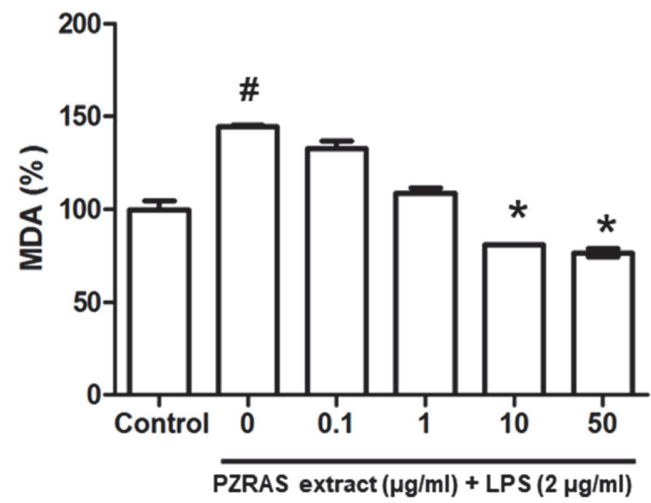

Fig. 2. Effect of PZRAS on malondialdehyde (MDA) in RAW264.7 cells. Cells were treated with or without PZRAS for $2 \mathrm{~h}$, and then incubated with LPS $(2 \mu \mathrm{g} / \mathrm{ml})$ for $20 \mathrm{~h}$. The values determined the means \pm SD of at least three independent experiments. ${ }^{*} p<0.05$ versus LPS-alone cells (\#). 
A

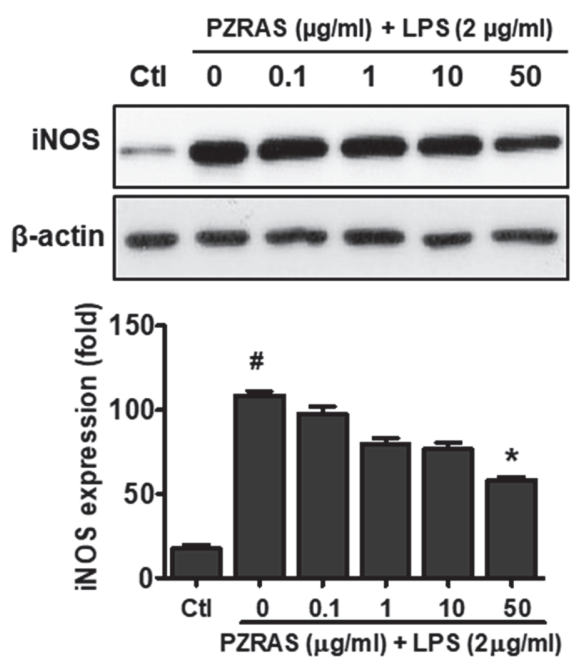

B

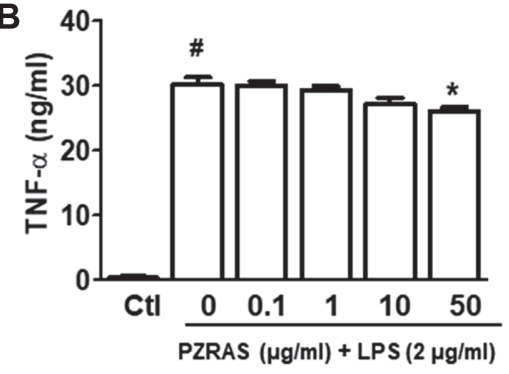

C

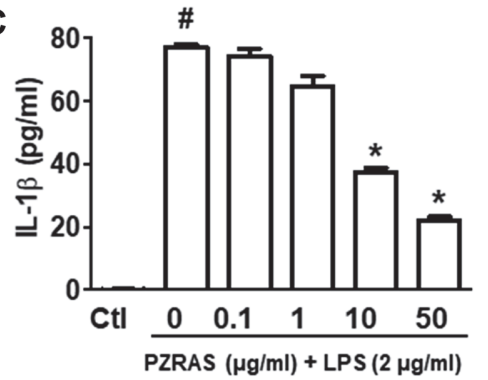

Fig. 3. Effects of PZRAS on anti-inflammatory activities in LPS-stimulated RAW264.7 cells. Cells were pretreated with PZRAS, and then stimulated with LPS $(2 \mu \mathrm{g} / \mathrm{ml})$ for $20 \mathrm{~h}$. (A) Protein level of iNOS was assayed by western blot analysis. The secreted levels of TNF- $\alpha$ (B) and IL-1 $\beta$ (C) were assessed by commercially available ELISA kit. Data represent the means \pm SD of at least three independent experiments. ${ }^{*} p<0.05$ versus LPS-alone cells (\#).

Effects of PZRAS on RANKL-Activated Osteoclast Differentiation and mRNA Expression in RAW264.7 Cells

Several pro-inflammatory cytokines including IL- $1 \beta$, TNF- $\alpha$, IL-6, IL-15, IL-17, and IL-18 were reported to regulate osteoclastogensis [37]. To examine the anti-osteoclast property of PZRAS, RAW264.7 cells were differentiated with RANKL and/or PZRAS. In the absence of PZRAS, RAW264.7 cells differentiated into mature TRAP-positive multinucleated cells (MNCs), but PZRAS inhibited the differentiation into matured MNCs (Fig. 4A). RANKL-RANK signaling is related with the elevation of osteoclastogenesis-related genes such as TRAP, cathepsin K, and MMP-2, -9 [38]. RT-PCR was used to verify the effects of PZRAS on RANKL-induced mRNA expression in RAW264.7 cells. The mRNA expressions of MMP-2, -9, TRAP, and cathepsin K were induced by RANKL. However, PZRAS at $50 \mu \mathrm{g} / \mathrm{ml}$ significantly suppressed this induction $(p<0.05)$ (Fig. 4B).

Effects of PZRAS on RANKL-Induced MAPK Expression in RAW264.7 Cells

The mitogen-activated protein kinases, ERK, JNK, and p38 were stimulated through RANKL-RANK signaling pathway in osteoclast precursor cells or RAW264.7 cells $[39,40]$. To clarify the intracellular mechanism of PZRAS in RANKL-signaling pathway, activities of ERK, JNK, and p38 were examined by western blot analysis. As shown in Fig. 5, RANKL at $50 \mathrm{ng} / \mathrm{ml}$ activated all three MAPKs in RAW264.7 cells, whereas PZRAS downregulated the activities of ERK and JNK but not p38 (Fig. 5).

\section{Effects of PZRAS on NFATc1 and c-Fos in RAW264.7 Cells}

The RANKL-RANK axis resulted in the activation of RANKL-induced transcription factors such as NFATc1 or c-Fos during osteoclast formation [41, 42]. In a next step, effects of PZRAS on the expressions of NFATc1 or c-Fos were investigated during osteocalstogenesis of RAW264.7 cells. RANKL upregulated mRNA expressions of NFATc1 or c-Fos compared with control group but $50 \mu \mathrm{g} / \mathrm{ml}$ of PZRAS significantly reduced those transcription activities (Fig. 6A). RANKL also increased the protein expressions of NFATc1 and c-Fos within $12 \mathrm{~h}$ in RAW264.7 cells. However, PZRAS inhibited those protein levels compared to untreated group (Fig. 6B). These results imply that PZRAS can prevent osteoclast maturation by inhibiting RANKL-induced NFATc1 and c-Fos.

\section{Effect of PZRAS on LPS-Treated Bone Lysis}

To examine the suppressive effect of PZRAS on bone lysis, 6-week-old mice were injected with LPS without or with different PZRAS. We also examined the efficiency of PZRAS for bone loss by comparing with an antioxidant complex $\left(85 \mathrm{mg} / \mathrm{kg}\right.$ vitamin D and $\left.1 \mu \mathrm{g} / \mathrm{ml} \mathrm{Ca}^{2+}\right)$. Micro-CT images revealed that LPS induced serious bone destruction in murine femurs. In contrast, PZRAS significantly diminished LPS-induced bone destruction at the indicated concentrations (Fig. 7A). The microstructural indices in femurs revealed that LPS injection significantly decreased bone volume/tissue volume (BV/TV) compared with vehicle group $(p<0.05)$. However, those reductions were markedly reversed by PZRAS at 200 or $400 \mathrm{mg} / \mathrm{kg}$ (Fig. 7B). H\&E stain revealed that osteoclast maturation and bone loss by LPS were significantly recovered by treatment with PZRAS. Although LPS significantly caused bone loss in the femurs, PZRAS protected them from bone loss (Fig. 7C). Although the parameter of eroded bone surface per bone surface (ES/BS) of femurs significantly increased in the LPS-injected 
A
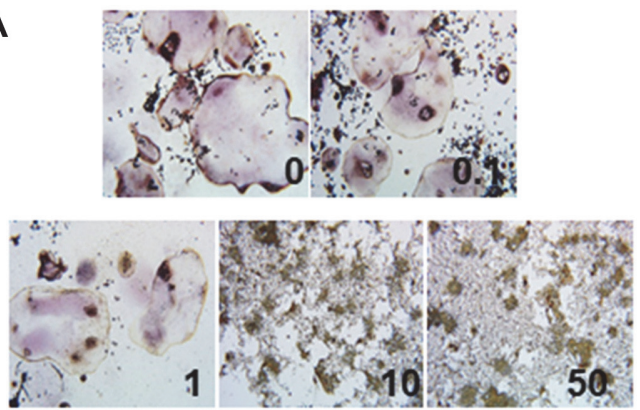

B

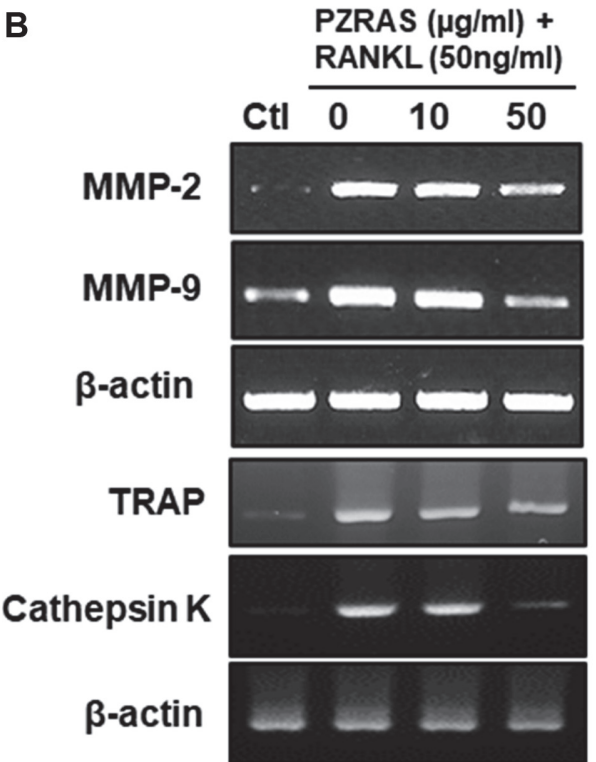

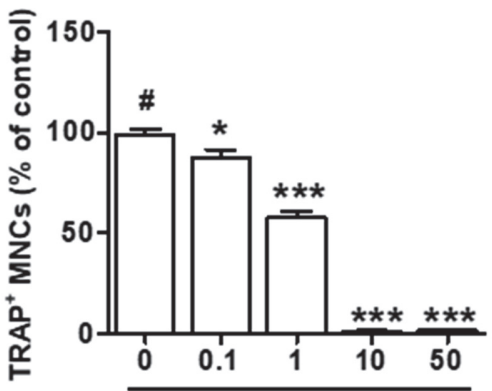

PZRA S $(\mu \mathrm{g} / \mathrm{ml})+$ RANKL $(50 \mathrm{ng} / \mathrm{ml})$

Fig. 4. TRAP staining and RT-PCR in RANKL-induced RAW264.7 cells treated with PZRAS. Cells were maintained with indicated concentrations of PZRAS in the presence of RANKL $(50 \mathrm{ng} / \mathrm{ml})$. (A) After 6 days, cells were stained and photographed, and the number of TRAP-positive multinucleated (TRAP ${ }^{+} \mathrm{MNCs}$ ) were counted (B) The mRNA expressions of MMP-2, MMP-9, TRAP, and cathepsin K were analyzed by RT-PCR. The values determined the means \pm SD of at least three independent experiments. ${ }^{*} p<0.05$ versus LPS-alone cells (\#).

RANKL (min) $\quad 0 \quad 5 \quad 15 \quad 30 \quad 0 \quad 5 \quad 1530$

PZRAS extract $(\mu \mathrm{g} / \mathrm{ml}) \quad$ - $\quad$ - $\quad$ - $\quad$ - $5050 \quad 5050$

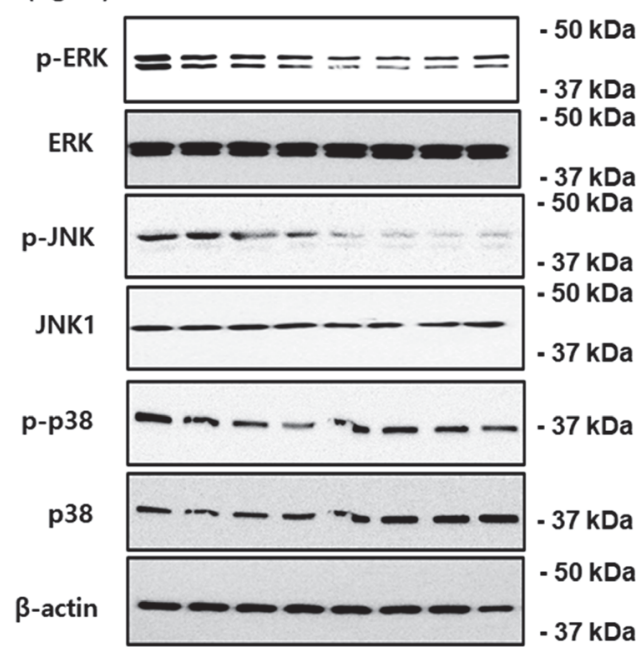

Fig. 5. Effects of PZRAS on MAPKs in RANKL-stimulated cells. RAW264.7 cells were pretreated with $50 \mu \mathrm{g} / \mathrm{ml}$ of PZRAS, and induced with RANKL $(50 \mathrm{ng} / \mathrm{ml})$ for indicated times. Expressed amounts of proteins were assessed by immunoblot against specific antibodies and normalized using $\beta$-actin. 
A

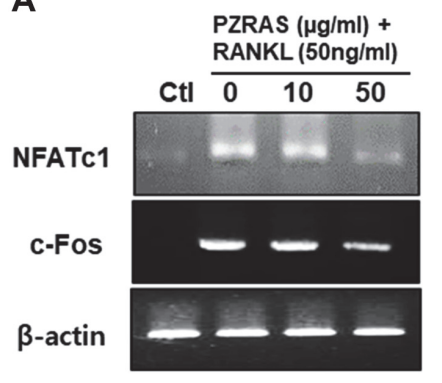

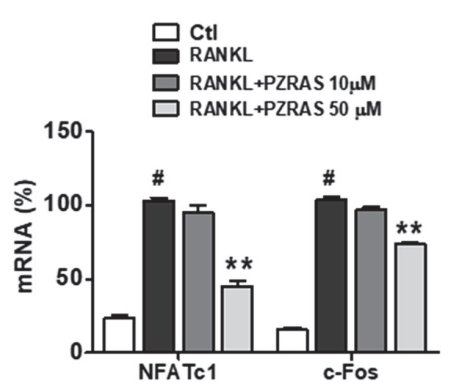

B

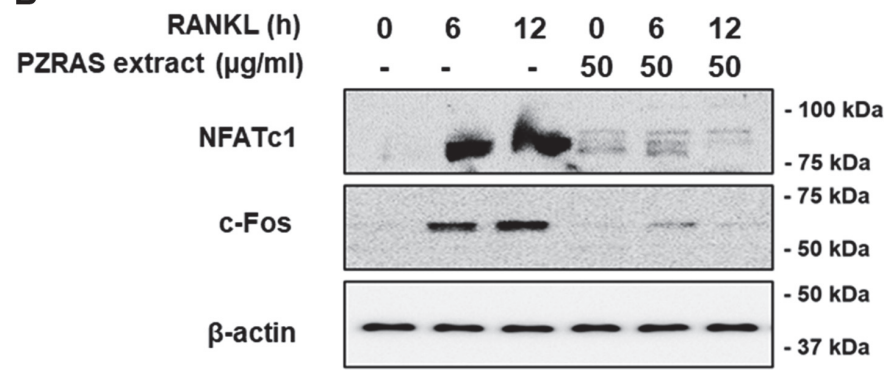

Fig. 6. Effects of PZRAS on NFATc1 and c-Fos in RANKL-stimulated cells. RAW264.7 cells were treated with PZRAS in the presence of RANKL (50 ng/ $\mathrm{ml}$ ). (A) The mRNA expressions of NFATc1 and c-Fos were analyzed by RT-PCR and the histogram exhibited the levels of mRNA (\%) of NFATc1 and c-Fos compared with control (\#). (B) The protein expressions of NFATc1 and c-Fos were analyzed by immunoblot against specific antibodies. ${ }^{* *} p<0.01$ versus LPS-only cells (\#).

A

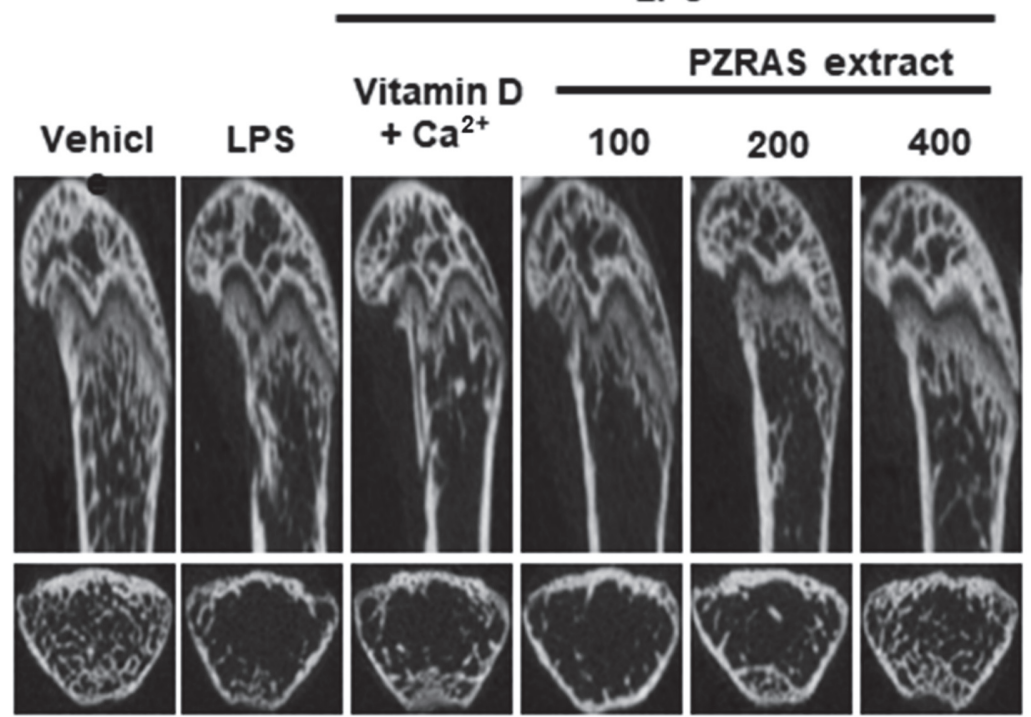

B

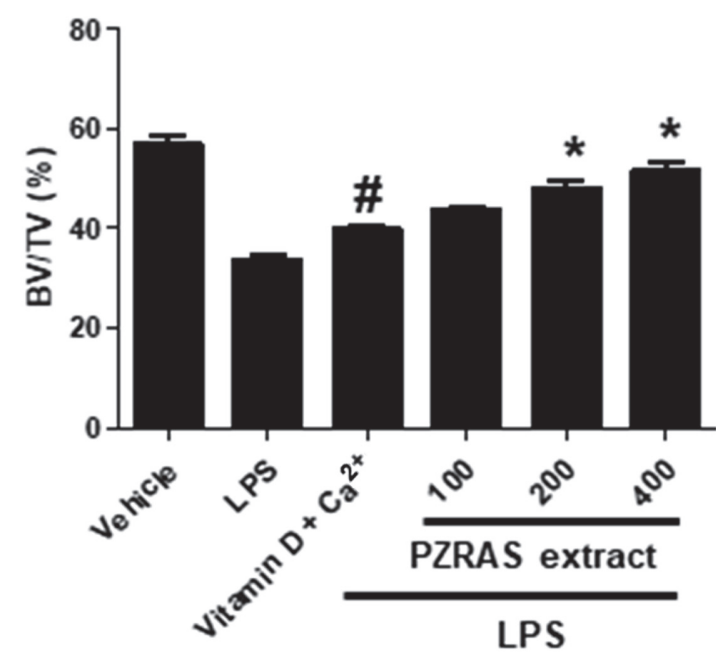

C

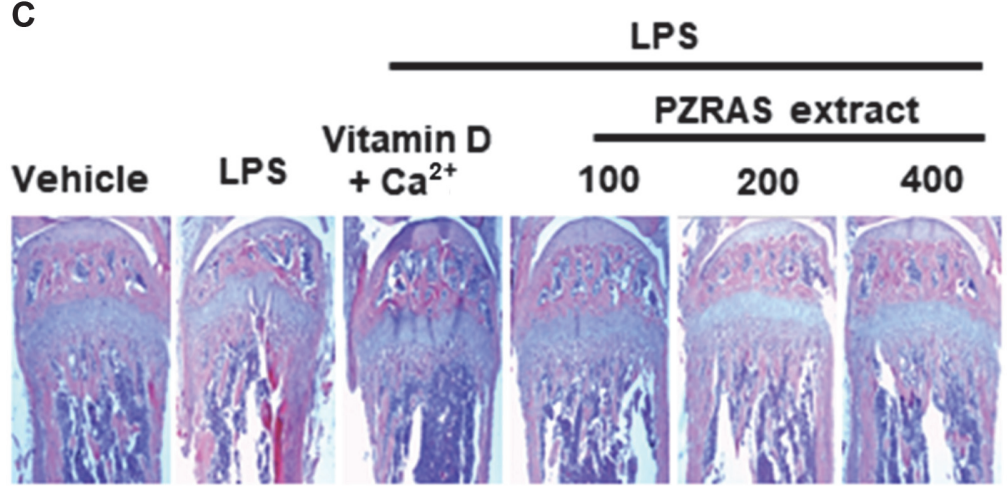

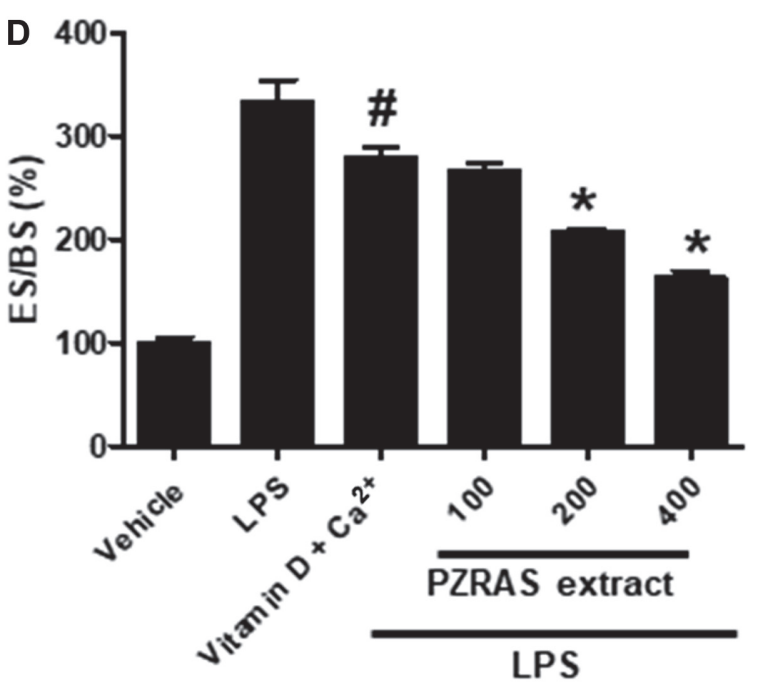

Fig. 7. Effects of PZRAS on LPS-mediated bone erosion. (A) Six-week-old ICR mice were injected intraperitoneally with or without LPS (5 mg/kg body weight). PZRAS was orally administered 1 day prior to LPS injection and every day thereafter for 14 days. Tissues and organs were collected and subjected to radiographs of the longitudinal and transverse section of the proximal femurs by micro CT. (B) The histogram represents the bone volume/tissue volume (BV/TV) of the femurs. The vitamin D $(85 \mathrm{mg} / \mathrm{kg})+\mathrm{Ca}^{2+}(1 \mu \mathrm{g} / \mathrm{ml})$ group was used as a positive control. (C) Histological analysis of murine femurs was carried out for bone morphology and osteoclast marker was detected by H\&E staining. (D) Eroded bone surface (BS) of the femurs near the growth plate was examined by histomorphometric analysis. ${ }^{*} p<0.05$ versus vitamin $\mathrm{D}+\mathrm{Ca}^{2+}(\#)$. 
mice compared to vehicle group $(p<0.05)$, this ratio was significantly recovered in PZRAS-treated mice $(p<0.05)$ (Fig. 7D). Taken together, the above results strongly suggest that PZRAS can inhibit osteoclast differentiation and bone loss.

\section{Discussion}

Natural flavonoid compounds are good targets of therapeutic agents because they are not associated with severe risk and possibility of long-term treatment. Therefore, the selection of natural compounds can be important to develop therapeutics for osteoporosis [7].

In this study, various compounds of flavonoids, P. ginseng, Z. jujube, Rubi fructus, A. asiaticae and S. baicalensis (PZRAS) were selected for anti-inflammatory activity and examined whether they had a protective effect against bone loss. Thus, we elucidated the anti-inflammatory activities of PZRAS in both in vitro and in vivo systems. The dual activities against iNOS and inflammation cytokine IL- $1 \beta$ were associated with increased antioxidant properties (CAT, SOD, GSH-Px and GSH-R). In addition, PZRAS has shown significant efficacy in protecting from LPS-induced bone loss, suggesting its potential therapeutic usage for bone disorders.

In previous studies, LPS can activate the productions of TNF- $\alpha$, IL- $1 \beta$, NO and other oxidative parameters [43, 44]. Moreover, abnormal expressions of TNF- $\alpha, \mathrm{IL}-1 \beta$, and NO are associated with chronic inflammatory diseases [45]. Therefore, the suppression of pro-inflammatory cytokines and regulators could be applied for treating immune disorders [36]. A recent study demonstrated that $P$. ginseng leaf extract including ginsenosides Rd and $\mathrm{Km}$ inhibited TNF- $\alpha$-enhanced expression of iNOS in HepG2 cells [46]. In this study, PZRAS dramatically repressed the expression of iNOS as well as secretion of IL-1 $\beta$ and TNF- $\alpha$ in LPS-stimulated RAW264.7 cells. These results suggest that PZRAS may exhibit the anti-inflammatory capacity through suppression of proinflammatory factors such as iNOS, IL-1 $\beta$ or TNF- $\alpha$.

Free radical attack on plasma membrane components such as LPS generates MDA. GSH is a strong antioxidant for cellular detoxification processes which can reduce MDA production. Therefore, it has been suggested that endogenous GSH plays an essential role in preventing oxidative stress-related inflammation [13]. In this study, PZRAS effectively increased activities of CAT, SOD, and GSH-Px and markedly decreased the MDA level (Table 2 and Fig. 2). These results suggest that PZRAS may act as an antioxidant agent via upregulation of CAT, SOD, and GSH-Px activities and suppression of MDA production.

Previous studies have demonstrated that the RANK/RANKL axis leads to activation of several downstream signaling pathways for osteoclast-specific genes and transcription factors [38-40]. For instance, MAPKs regulate NFATcl and c-Fos, transcription factors which then modulate the MMP-9 mRNA expression during osteoclast maturation $[41,42]$. PZRAS significantly inhibited phosphorylated ERK and JNK, (but not p38 expression), mRNA expressions of MMP-2, -9, TRAP, cathepsin K as well as transcription factors (NFATc1, c-Fos) during osteoclast differentiation. We also demonstrated that PZRAS efficiently worked to cure bone loss in LPS-induced animal model as evidenced by its improving BV/TV and ES/BS.

The above results clearly show that PZRAS exhibits an anti-osteoclastogenic potential by reducing the generation of iNOS, IL-1 $\beta$, and MMP-2, -9, and also increased levels of antioxidant enzymes (CAT, SOD, GSH-Px and GSH reductase). PZRAS also prevented LPS-induced bone destruction in mice. Therefore, our findings suggest that PZRAS could be a promising therapeutic candidate for various bone-related diseases.

\section{Acknowledgment}

This work was funded by a grant from the Ministry of Knowledge Economy, Republic of Korea (R0000949).

\section{Conflict of Interest}

The authors have no financial conflicts of interest to declare.

\section{References}

1. Ralston SH, de Crombrugghe B. 2006. Genetic regulation of bone mass and susceptibility to osteoporosis. Genes Dev. 20: $2492-2506$.

2. McGreevy C, Williams D. 2011. Safety of drugs used in the treatment of osteoporosis. Ther. Adv. Drug Saf. 2: 159-172.

3. O'Regan RM, Gradishar WJ. 2001. Selective estrogen-receptor modulators in 2001. Oncology (Williston Park, NY) 15: 1177-1185.

4. Rejnmark L, Mosekilde L. 2011. New and emerging antiresorptive treatments in osteoporosis. Curr. Drug Saf. 6: 75-88.

5. Khosla S, Westendorf JJ, Oursler MJ. 2008. Building bone to reverse osteoporosis and repair fractures. J. Clin. Invest. 118: 421-428.

6. Jung HW, Seo UK, Kim JH, Leem KH, Park YK. 2009. Flower extract of Panax notoginseng attenuates lipopolysaccharide-induced inflammatory response via blocking of NF-kappaB signaling pathway in murine macrophages. J. Ethnopharmacol. 122: 313-319.

7. Jia M, Nie Y, Cao DP, Xue YY, Wang JS, Zhao L, et al. 2012. Potential antiosteoporotic agents from plants: a comprehensive review. Evid. Based Complement. Alternat. Med. 2012: 364604.

8. Adegbola P, Aderibigbe I, Hammed W, Omotayo T. 2017. Antioxidant and anti-inflammatory medicinal plants have potential role in the treatment of cardiovascular disease: a review. Am. J. Cardiovasc. Dis. 7: 19-32.

9. Chen L, Deng H, Cui H, Fang J, Zuo Z, Deng J, et al. 2018. Inflammatory responses and inflammation-associated diseases in organs. Oncotarget 9: 7204-7218.

10. Kang HS, Lee JY, Kim CJ. 2008. Anti-inflammatory activity of arctigenin from Forsythiae Fructus. J. Ethnopharmacol. 116: 305-312.

11. Cui XY, Kim JH, Zhao X, Chen BQ, Lee BC, Pyo HB, et al. 2006. Antioxidative and acute anti-inflammatory effects of Campsis grandiflora flower. J. Ethnopharmacol. 103: 223-228.

12. Zhang XY, Tan YL, Cao LY, Wu GY, Xu Q, Shen Y, et al. 2005. Antioxidant enzymes and lipid peroxidation in different forms of schizophrenia treated with typical and atypical antipsychotics. Schizophr. Res. 81: 291-300.

13. Sontakke AN, Tare RS. 2002. A duality in the roles of reactive oxygen species with respect to bone metabolism. Clin. Chim. Acta 318: $145-148$

14. Nishihara T, Koseki T. 2000. Microbial etiology of periodontitis. Periodontol. 2000 36: 14-26. 
15. Feng Z, Weinberg A. 2006. Role of bacteria in health and disease of periodontal tissues. Periodontol 2000 40: 50-76.

16. Guan SM, Shu L, Fu SM, Liu B, Xu XL, Wu JZ. 2009. Prevotella intermedia upregulates MMP-1 and MMP-8 expression in human periodontal ligament cells. FEMS Microbiol. Lett. 299: 214-222.

17. Visse R, Nagase H. 2003. Matrix metalloproteinases and tissue inhibitors of metalloproteinases: structure, function, and biochemistry. Circ. Res. 92: 827-839.

18. Sorsa T, Tjaderhane L, Konttinen YT, Lauhio A, Salo T, Lee HM, et al. 2006. Matrix metalloproteinases: contribution to pathogenesis, diagnosis and treatment of periodontal inflammation. Ann. Med. 38: 306-321.

19. Islam S, Hassan F, Tumurkhuu G, Dagvadorj J, Koide N, Naiki Y, et al. 2007. Bacterial lipopolysaccharide induces osteoclast formation in RAW 264.7 macrophage cells. Biochem Biophys. Res. Commun. 360: 346-351.

20. Smith BJ, Lerner MR, Bu SY, Lucas EA, Hanas JS, Lightfoot SA, et al. 2006. Systemic bone loss and induction of coronary vessel disease in a rat model of chronic inflammation. Bone 38: 378-386.

21. Itoh K, Udagawa N, Kobayashi K, Suda K, Li X, Takami M, et al. 2003. Lipopolysaccharide promotes the survival of osteoclasts via Toll-like receptor 4, but cytokine production of osteoclasts in response to lipopolysaccharide is different from that of macrophages. J. Immunol. 170: 3688-3695.

22. Zhang G, Liu A, Zhou Y, San X, Jin T, Jin Y. 2008. Panax ginseng ginsenoside-Rg2 protects memory impairment via anti-apoptosis in a rat model with vascular dementia. J. Ethnopharmacol. 115: 441-448.

23. Cho YS, Kim CH, Ha TS, Lee SJ, Ahn HY. 2013. Ginsenoside rg2 inhibits lipopolysaccharide-induced adhesion molecule expression in human umbilical vein endothelial cell. Korean J. Physiol. Pharmacol. 17: 133-137.

24. Zhang H, Jiang L, Ye S, Ye Y, Ren F. 2010. Systematic evaluation of antioxidant capacities of the ethanolic extract of different tissues of jujube (Ziziphus jujuba Mill.) from China. Food Chem. Toxicol. 48: 1461-1465.

25. He JM, Chen SC, Li RP, Yuan LX, Bao JM, Guo ML. 2015. Suppression of nuclear factor-kappa B and mitogen-activated protein kinase signalling pathways by goshonoside-F5 extracted from Rubi Fructus. Int. Immunopharmacol. 24: 182-190.

26. Diaz P, Jeong SC, Lee S, Khoo C, Koyyalamudi SR. 2012. Antioxidant and anti-inflammatory activities of selected medicinal plants and fungi containing phenolic and flavonoid compounds. Chin. Med. 7: 26.

27. Wang S, Li J, Sun J, Zeng KW, Cui JR, Jiang Y, et al. 2013. NO inhibitory guaianolide-derived terpenoids from Artemisia argyi. Fitoterapia 85: 169-175.

28. Wong RW, Rabie AB. 2006. Traditional Chinese medicines and bone formation-a review. J. Oral Maxillofac. Surg. 64: 828-837.

29. Huet O, Petit JM, Ratinaud MH, Julien R. 1992. NADH-dependent dehydrogenase activity estimation by flow cytometric analysis of 3-(4,5-dimethylthiazolyl-2-yl)-2,5-diphenyltetrazolium bromide (MTT) reduction. Cytometry 13: 532-539.

30. Prasad K, Mantha SV, Muir AD, Westcott ND. 2000. Protective effect of secoisolariciresinol diglucoside against streptozotocininduced diabetes and its mechanism. Mol. Cell Biochem. 206: 141-149.

31. Aebi H. Catalase in vitro. 1984. Methods Enzymol. 105: 121-126.

32. Choi HJ, Eun JS, Park YR, Kim DK, Li R, Moon WS, et al. 2008. Ikarisoside A inhibits inducible nitric oxide synthase in lipopolysaccharide-stimulated RAW 264.7 cells via p38 kinase and nuclear factor-kappaB signaling pathways. Eur. J. Pharmacol. 601: 171-178.

33. Choi HJ, Park YR, Nepal M, Choi BY, Cho NP, Choi SH, et al. 2010. Inhibition of osteoclastogenic differentiation by Ikarisoside A in RAW 264.7 cells via JNK and NF-kappaB signaling pathways. Eur. J. Pharmacol. 636: 28-35.

34. Nepal M, Choi HJ, Choi BY, Yang MS, Chae JI, Li L, et al. 2013. Hispidulin attenuates bone resorption and osteoclastogenesis via the RANKL-induced NF-kappaB and NFATc1 pathways. Eur. J. Pharmacol. 715: 96-104.

35. Luo A, Ge Z, Fan Y, Luo A, Chun Z, He X. 2011. In vitro and in vivo antioxidant activity of a water-soluble polysaccharide from Dendrobium denneanum. Molecules 16: 1579-1592.

36. Arango Duque G, Descoteaux A. 2014. Macrophage cytokines: involvement in immunity and infectious diseases. Front. Immunol. 5: 491

37. Wojdasiewicz P, Poniatowski LA, Szukiewicz D. 2014. The role of inflammatory and anti-inflammatory cytokines in the pathogenesis of osteoarthritis. Mediators Inflamm. 2014: 561459.

38. Sundaram K, Nishimura R, Senn J, Youssef RF, London SD, Reddy SV. 2007. RANK ligand signaling modulates the matrix metalloproteinase-9 gene expression during osteoclast differentiation. Exp. Cell Res. 313: 168-178.

39. Lee KH, Seo IC, Choi MH, Jeong DW. 2018. Roles of mitogen-activated protein kinases in osteoclast biology. Int. J. Mol. Sci. 19: 30043024 .

40. Raphael H, Eric J, Rajaram G, Kim CM. 2019. Bone morphogenetic proteins: Their role in regulating osteoclast differentiation. Bone Rep. 10: 100207-100216.

41. Suda T, Nakamura I, Jimi E, Takahashi N. 1997. Regulation of osteoclast function. J. Bone Miner. Res. 12: 869-79.42.

42. Wong BR, Josien R, Lee SY, Vologodskaia M, Steinman RM, Choi Y. 1998. The TRAF family of signal transducers mediates NF- $\kappa B$ activation by the TRANCE receptor. J. Biol. Chem. 273: 28355-28359.

43. Sheeba MS, Asha VV. 2009. Cardiospermum halicacabum ethanol extract inhibits LPS induced COX-2, TNF-alpha and iNOS expression, which is mediated by NF-kappa B regulation, in AW264.7 cells. J. Ethnopharmacol. 124: 39-44.

44. Park JY, Cho HY, Kim JK, Noh KH, Yang JR, Ahn JM, et al. 2005. Chlorella dichloromethane extract ameliorates NO production and iNOS expression through the down-regulation of NF kappa B activity mediated by suppressed oxidative stress in RAW 264.7 macrophages. Clin. Chim. Acta 351: 185-196.

45. Soufli I, Toumi R, Rafa H, Touil-Boukoffa C. 2016. Overview of cytokines and nitric oxide involvement in immuno-pathogenesis of inflammatory bowel diseases. World J. Gastrointest. Pharmacol. Ther. 6: 353-360.

46. Song SB, Tung NH, Quang TH, Ngan NT, Kim KE, Kim YH. 2012. Inhibition of TNF-alpha-mediated NF-kappaB transcriptional activity in HepG2 cells by dammarane-type sponins from Panax ginseng leaves. J. Ginseng Res. 36: 146-152.

47. Balli U, Cetinkaya BO, Keles GC, Keles ZP, Guler S, Sogut MU, et al. 2016. Assessment of MMP-1, MMP-8 and TIMP-2 in experimental periodontitis treated with kaempferol. J. Periodontal Implant Sci. 46: 84-95. 